\title{
Cetuximab intensifies the ADCC activity of adoptive NK cells in a nude mouse colorectal cancer xenograft model
}

\author{
SHANSHAN CHEN ${ }^{1}$, XUECHUN LI $^{2}$, RONGMING CHEN $^{1}$, MINGANG YIN $^{1}$ and QIUHONG ZHENG ${ }^{1}$ \\ ${ }^{1}$ Fujian Provincal Key Laboratory of Tumor Biotherapy, Teaching Hospital of Fujian Medical University, \\ Fujian Provincal Cancer Hospital; ${ }^{2}$ Provincial Clinical College of \\ Fujian Medical University, Fuzhou, Fujian 350014, P.R. China
}

Received May 10, 2015; Accepted June 21, 2016

DOI: $10.3892 / \mathrm{ol} .2016 .4835$

\begin{abstract}
Natural killer (NK) cells, discovered $\sim 40$ years ago, are believed to be the most effective cytotoxic lymphocytes to counteract cancer; however, adoptive NK cell therapy in vivo has encountered certain limitations, including a lack of specificity. The drug cetuximab can mediate antibody dependent cell mediated cytotoxicity (ADCC) activity through NK cells in vivo, and has been approved for the first-line treatment of epidermal growth factor receptor (EGFR)-positive metastatic colorectal cancer (CRC). However, the ADCC activity of adoptive NK cells, induced by cetuximab in a nude mouse CRC xenograft model, has not been previously reported. The aim of the present study was to explore the ADCC activity of cetuximab combined with adoptive NK cells in CRC xenograft models with various EGFR expressions. The nude mouse xenograft models were established by subcutaneously injecting LOVO or SW620 cells. The mice were then randomly divided into 6 groups: Phosphate-buffered saline, cetuximab, human immunoglobulin G (hIgG), NK cells, hIgG plus NK cells and cetuximab plus NK cells. The ADCC antitumor activity was evaluated in these CRC models. The results indicated that the cetuximab plus NK cells group showed the greatest tumor inhibition effect compared with the NK cells group in LOVO xenograft tumor models with positive EGFR expression. However, the combination of cetuximab and NK cells did not show a stronger tumor inhibitory effect against the SW620 xenograft tumor models compared with the efficiency of NK cells. In conclusion, cetuximab could intensify the ADCC antitumor activity of adoptive NK cells towards CRC with an increased EGFR expression. The combination of cetuximab
\end{abstract}

Correspondence to: Professor Qiuhong Zheng, Fujian Provincal Key Laboratory of Tumor Biotherapy, Teaching Hospital of Fujian Medical University, Fujian Provincal Cancer Hospital, 420 Fuma Road, Fuzhou, Fujian 350014, P.R. China

E-mail:zqh2858@foxmail.com

Key words: colorectal cancer, cetuximab, natural killer cells, epidermal growth factor receptor, antibody dependent cell mediated cytotoxicity activity and NK cells may be a potential immunotherapy for metastatic CRC patients with positive EGFR expression.

\section{Introduction}

Colorectal cancer (CRC) is the fifth leading cause of cancer-associated mortality in China (1). Surgery is the first-line treatment for patients with early-stage and localized tumors, but certain patients with recurrent or metastatic disease are inoperable when they arrive at the hospital. Chemotherapy and radiotherapy could be adopted for such patients, yet the adverse effects can be unbearable and the 5-year survival rate remains low (2). Therefore, novel treatment approaches with acceptable toxicity levels are required to improve the therapeutic efficacy and survival rate of inoperable patients.

Since the 1990s, certain novel achievements towards improving the prognosis of patients with advanced CRC were established, including an immunotherapy strategy based on antigen-targeted therapy via monoclonal antibodies (mAbs). mAbs, including panitumumab, bevacizumab and cetuximab, have been recently approved by the Food and Drug Administration for first-line treatment of advanced CRC (3). In addition, the adoptive transfer of natural killer (NK) cells is also an emerging method to eliminate the metastasis of CRC $(4,5)$.

Cetuximab, a human-mouse chimerized anti-epidermal growth factor receptor (EGFR) immunglobulin G1 (IgG1) monoclonal antibody, has been previously used to specifically combine with EGFR on the cell surface and intercept downstream signal conduction; as a result, tumor cell proliferation, invasion, metastasis, angiogenesis were inhibited and tumor cell apoptosis were promoted (6-8). In addition, cetuximab may target tumor cells by antibody dependent cell mediated cytotoxicity (ADCC) activity (9). However, data accumulated from clinical studies indicate that metastatic CRC showed limited responses to cetuximab, when used as a monotherapy (10).

The use of NK cells, the most powerful type of innate immune cell, is another immunotherapy option for patients with advanced CRC. NK cells were previously demonstrated to directly kill human tumor cells that had been freshly isolated from gastric, renal cell and colon carcinomas (11). The cytotoxicity of NK cells is regulated by a series of activating or inhibiting signals, which may improve the sensitivity of NK cells to affecting tumor cells $(9,12,13)$. Activated NK cells 
can kill tumor cells directly through the release of intracellular toxic substances, including perforin and granzyme (14). Furthermore, NK cells produce cytokines, such as interferon- $\gamma$ (IFN- $\gamma$ ), tumor necrosis factor- $\alpha$ and interleukin-2 (IL-2), to enhance the antitumor effects and mediate tumor cell apoptosis via the FasL/Fas apoptosis pathway $(15,16)$. NK cells can also induce ADCC activity in order to lyse tumor cells (14). Although the results of NK cell therapy have been promising in vitro, adoptive NK cell therapy in vivo possesses certain limitations, including the immunosuppressive environment of CRC or a lack of specificity (17).

ADCC activity is regulated by a complex balance of activating and inhibiting signals, including membrane coreceptors and serum proteins, such as cytokines, chemokines and hormones (18). NK cells only express activated cluster of differentiation (CD)16a (also called Fc fragment of IgG receptor IIIa), and are widely accepted as the key immune cells to mediate ADCC antitumor activity (19). The Fc receptor of NK cells can identify the constant region of tumor-bound antibodies, which allows NK cells to kill antibody-coated target cells via ADCC activity specifically (20). Siokawa et al (15) confirmed that rituximab could significantly enhance the ADCC activity and improve the killing effects of NK cells towards leukemia xenograft cells in immune deficient NOG mice. Elotuzumab, a monoclonal antibody, was shown to enhance NK cell-mediated ADCC activity in SLAM family member 7-expressing myeloma cells (21). Roda et al (22) found that after combining with cetuximab, NK cells would enhance IFN- $\gamma$ secretion by 3-10 times. These studies demonstrate that the combination of NK cell therapy with antibody-based immunotherapy may be an effective way to enhance the antitumor activity towards CRC.

In a previous study, the ADCC activity of NK cells was demonstrated to be important in cetuximab-induced cytotoxicity in EGFR-positive colon cancer cells in vitro (23). In addition, Yang et al (24) suggested that cetuximab could mediate ADCC activity through NK cells in vivo. However, the ADCC activity of adoptive NK cells, induced by cetuximab in a nude mouse CRC xenograft model, has not been previously reported. In the present study, healthy human NK cells were regarded as effector cells, and the ADCC activity or antitumor effects of cetuximab combined with adoptive NK cells were observed in CRC xenograft models with varying degrees of EGFR expression. The present study was conducted in order to explore a potential immunotherapy for advanced CRC, based on the combination of cetuximab and adoptive NK cells.

\section{Materials and methods}

Animals and cell lines. In total, $60 \mathrm{BALB} / \mathrm{c}$ nude mice (female; weight, 17-18 g; age, 4-5 weeks) were purchased from the Shanghai Laboratory Animal Center Laboratory Animal Co., Ltd. [Shanghai, China; animal quality certificate code, SCXK (Shanghai) 2007-000528569]. All mice were bred in specific pathogen-free (SPF) conditions at the Fujian Medical University [Fuzhou, China; environmental license, SYXK (Fujian) 2008-0001], and were housed at constant temperature $\left(24 \pm 2^{\circ} \mathrm{C}\right)$ and $60 \%$ relative humidity, with a $10: 14 \mathrm{~h}$ light-dark cycle. Mice had ad libitum access to food and autoclaved water. All the animal procedures were approved by the Animal
Ethics Committee of Fujian Medical University (Fuzhou, China). LOVO and SW620 cell lines were obtained from the Cell Bank of Type Culture Collection of Chinese Academy of Sciences (Shanghai, China).

Antibodies and reagents. Cetuximab was purchased from Merck Millipore (Darmstadt, Germany). The antibodies used in the present study were mouse anti-human CD3-fluorescein isothiocyanate (FITC; monoclonal; 1:100; cat. no. 55539; BD Biosciences, Franklin Lakes, NJ, USA), mouse anti-human CD56-phycoerythrin (PE) (monoclonal; 1:100; cat. no. 555516; BD Biosciences); human immunoglobulin G (hIgG; polyclonal; 1:200; cat. no. bs-0297P; Beijing Biosynthesis Biotechnology Co., Ltd., Beijing, China), rabbit anti-human EGFR (polyclonal; 1:200; cat. no. Sc-03AC; Santa Cruz Biotechnology, Inc.) rabbit anti-human Ki-67 (monoclonal; 1:200; cat. no ZA-0502; OriGene Technologies, Inc., Beijing, China). The PV-9000 polymer detection system for immunohistological staining, apoptosis detection kits in situ and diaminobenzidine color reagent were purchased from OriGene Technologies, Inc. The WST-1 cell proliferation reagent was purchased from Roche Diagnostics (Basel, Switzerland). RPMI-1640 and $0.25 \%$ ethylenediaminetetraacetic acid pancreatin were purchased from Takara Bio, Inc. (Otsu, Japan), and Ficoll-paque lymphocyte separation medium was purchased from GE Healthcare Life Sciences (Chalfont, UK). Recombinant IL-2 was purchased from Beijing Four Rings Biopharmaceutical Co., Ltd. (Beijing, China). Cells were analyzed by Moflow XDP flow cytometry with Summit version 5.2 software (Beckman Coulter, Inc., Brea, CA, USA).

Cancer cells culture. Human CRC SW620 and LOVO cells were cultured in RPMI-1640 medium containing $10 \%$ fetal bovine serum (FBS; Gibco; Thermo Fisher Scientific, Inc., Waltham, MA, USA) in an incubator at $37^{\circ} \mathrm{C}$ with $5 \% \mathrm{CO}_{2}$.

NK cell isolation and cultivation. A $40 \mathrm{ml}$ sample of peripheral blood was obtained from five healthy human donors between April and August 2013, which was approved by the Ethics Committee of Fujian Provincal Cancer Hospital (Fuzhou, China) and written informed consent was obtained. Peripheral blood mononuclear cells (PBMCs) were isolated using Ficoll-paque lymphocyte separation medium and then washed twice with phosphate-buffered saline (PBS). The PBMCs were resuspended in $100 \mu \mathrm{l}$ PBS, stained with $10 \mathrm{ul} \mathrm{CD3-FITC} \mathrm{and}$ 10 ul CD56-PE monoclonal antibodies and then incubated at $4^{\circ} \mathrm{C}$ for $30 \mathrm{~min}$ in darkness. The cells were then washed twice using PBS, prior to being evaluated by the MoflowXDP flow cytometry and sorted into $\mathrm{CD} 3^{-} \mathrm{CD} 56^{+} \mathrm{NK}$ cells. The NK cells were cultured in RPMI-1640 medium containing $20 \%$ fetal bovine serum, recombinant IL-2 (1,000 units (U)/ml), streptomycin $(100 \mu \mathrm{g} / \mathrm{ml})$ and penicillin $(100 \mathrm{U} / \mathrm{ml})$ for 10 days.

ADCC activity assay of NK cells in vitro. After 14 days of culture, NK cells were analyzed and collected. A small portion of NK cells $\left(\sim 10^{6}\right.$ cells) were analyzed by Moflow XDP flow cytometry, while the remaining NK cells were washed twice with PBS and resuspended at a density of $4 \times 10^{4}$ cells $/ \mathrm{ml}$ in RPMI-1640 medium containing 10\% FBS. LOVO and SW620 cells $\left(10^{4}\right.$ cells) were seeded in a 96-well plate for $24 \mathrm{~h}$ and 
Table I. Antibody dependent cell mediated cytotoxicity activity of natural killer cells towards LOVO and SW620 cells in vitro, expressed as the inhibition rate of LOVO or SW620 cells (\%).

Effector-target ratios

\begin{tabular}{lccrr}
\cline { 2 - 5 } Cell line & $40: 1$ & $20: 1$ & $10: 1$ & $5: 1$ \\
\hline LOVO & $52.14 \pm 1.45$ & $29.91 \pm 1.03$ & $19.62 \pm 0.78$ & $8.80 \pm 1.38$ \\
SW620 & $20.34 \pm 0.88$ & $14.05 \pm 1.10$ & $5.02 \pm 0.75$ & $3.07 \pm 0.45$
\end{tabular}

Values are presented as mean \pm standard error of the mean.

then cocultured with NK cells for $48 \mathrm{~h}$ at effector-to-target ratios of 40:1, 20:1, 10:1 and 5:1. Following coculture, the cells were marked by incubating with WST-1 for $4 \mathrm{~h}$ at $37^{\circ} \mathrm{C}$ in $5 \% \mathrm{CO}_{2}$. The lysis of cells was measured by examining optical density (OD) at $450 \mathrm{~nm}$, and the ADCC activity of NK cells was calculated using the following formula: Killing effect $(\%)=[1-(\mathrm{OD}$ value of experimental group - OD value of effect cells)/OD of target cells] x 100.

Establishment of nude mouse xenograft model. After one week of breeding in the SPF conditions, logarithmic phase LOVO or SW620 cells $\left(5 \times 10^{6}\right.$ cells) were subcutaneously injected into the mice via the left axillary (LOVO group, $n=30$; SW620 group, $n=30$ ). Tumorigenicity was observed, by assessing the tumor volume over time.

Experimental groups and intervention. When the volume of the tumors had reached $200 \mathrm{~mm}^{3}$ (7-8 $\mathrm{mm}$ in diameter), the 30 mice were divided into 6 groups and received the following interventions: i) Intraperitoneal injection of $0.2 \mathrm{ml} \mathrm{PBS}$; ii) intraperitoneal injection of cetuximab $(1 \mathrm{mg} / \mathrm{kg})$; iii) intraperitoneal injection of hIgG (1 mg/kg); iv) intravenous injection of NK cells ( $2 \times 10^{6}$ cells) through the caudal vein; v) intraperitoneal injection of $\mathrm{hIgG}(1 \mathrm{mg} / \mathrm{kg})$ and intravenous injection of NK cells $\left(2 \times 10^{6}\right)$; or vi) intraperitoneal injection of cetuximab $(1 \mathrm{mg} / \mathrm{kg})$ and intravenous injection of NK cells $\left(2 \times 10^{6}\right)$. These interventions were executed 3 times per week, for 4 weeks.

Nude mouse xenograft tumor assay. To observe the tumor growth in the mice, tumor diameter was measured 2 times per week using vernier calipers, and tumor volume was calculated with the formula: Volume $=0.5 \mathrm{x}$ long diameter $\mathrm{x}$ (short diameter $)^{2}$. Subsequent to data collection, a tumor growth curve was drawn. All the mice sacrificed by $\mathrm{CO}_{2}$ inhalation 3 days following the end of treatment and tumors were resected and weighed. The antitumor rate was evaluated with the formula: Antitumor rate $(\%)=$ (average tumor weight of control group - average tumor weight of experimental group)/average tumor weight of control group x 100. Pathological changes to the tumor tissues were observed by immunohistochemistry (IHC) and terminal deoxynucleotidyl transferase dUTP nick end labeling (TUNEL). IHC was used to detect the expression of EGFR and Ki-67, whereas xenograft cell apoptosis was analysed by TUNEL. Human CRC specimens obtained from CRC patients who provided written informed consent at Fujian Provincal Cancer Hospital (Fuzhou, China) in September 2013 were used as positive controls, while PBS was used as a

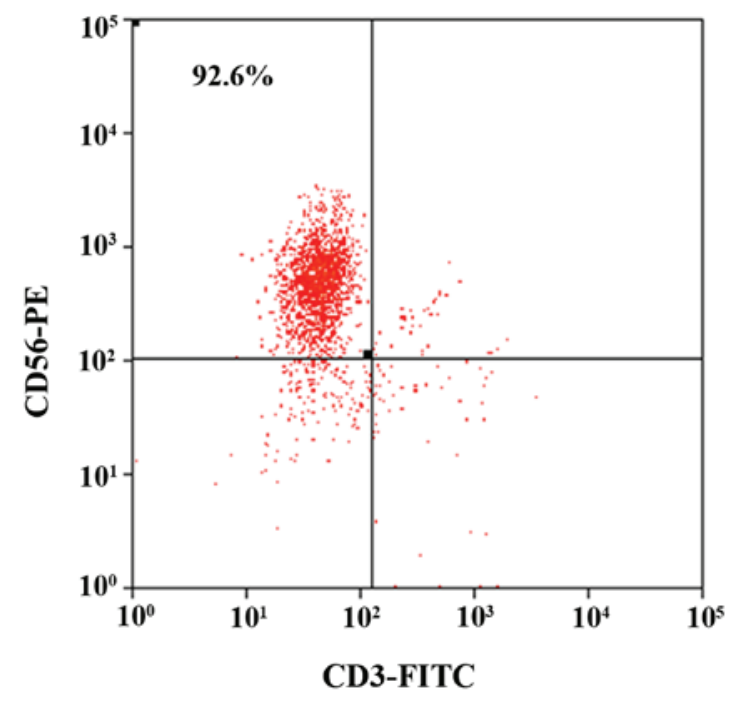

Figure 1. The ratio of $\mathrm{CD} 3 \mathrm{CD}^{-} 6^{+}$natural killer cells after 14 days of culture. CD, cluster of differenatiation; FITC, fluorescein isothiocyanate; $\mathrm{PE}$, phycoerythrin.

negative control. The expression level of EGFR or Ki-67 was assessed by randomly selecting 5 non-overlap ping fields of view in an optical microscope, and counting 100 tumor cells per field. The percentage of positive cells or apoptosis cells was regarded as the labeling index (LI) or apoptosis index (AI), respectively. An expression score of $0-10 \%$ was regarded as negative (-), 10-25\% as weak positive (+), 26-50\% as positive $(++)$ and $50-100 \%$ as strong positive $(+++)$.

Statistical analysis. SPSS 19.0 (IBM SPSS, Armonk, NY, USA) was used for the analysis of all statistics. Data were presented as the mean \pm standard error of the mean. One-way analysis of variance was used to determine the differences between multiple groups. Various groups were compared using the Bonferroni test. $\mathrm{P}<0.05$ was considered to indicate a statistically significant difference.

\section{Results}

Purity of NK cells. After 14 days of culture, the percentage of NK cells $\left(\mathrm{CD}^{-} \mathrm{CD}^{-} 6^{+}\right)$cells was $92.60 \pm 3.83 \%$ (Fig. 1$)$, which met the requirements for the following experiments.

ADCC activity of NK cells in vitro. The inhibition rate of CRC cells is shown on Table I. NK cells had a marked inhibitory 
Table II. Inhibitory rates of tumor weight in xenografts.

\begin{tabular}{|c|c|c|c|c|}
\hline \multirow[b]{2}{*}{ Group } & \multicolumn{2}{|c|}{ LOVO xenografts } & \multicolumn{2}{|c|}{ SW620 xenografts } \\
\hline & Weight, g & Inhibitory rate, $\%$ & Weight, g & Inhibitory rate, $\%$ \\
\hline Phosphate-buffered saline & $1.92 \pm 0.11$ & - & $1.68 \pm 0.05$ & - \\
\hline hIgG & $1.81 \pm 0.06$ & 5.73 & $1.73 \pm 0.06$ & -3.00 \\
\hline Cetuximab & $1.37 \pm 0.05$ & 28.65 & $1.63 \pm 0.03$ & 3.00 \\
\hline NK cells & $1.36 \pm 0.09$ & 29.17 & $1.23 \pm 0.06$ & 26.79 \\
\hline hIgG+NK cells & $1.38 \pm 0.07$ & 28.13 & $1.35 \pm 0.04$ & 19.64 \\
\hline Cetuximab+NK cells & $1.00 \pm 0.05$ & 47.92 & $1.29 \pm 0.05$ & 23.21 \\
\hline
\end{tabular}

Values presented as mean \pm standard error of the mean. hIgG, human immunoglobulin G; NK, natural killer.
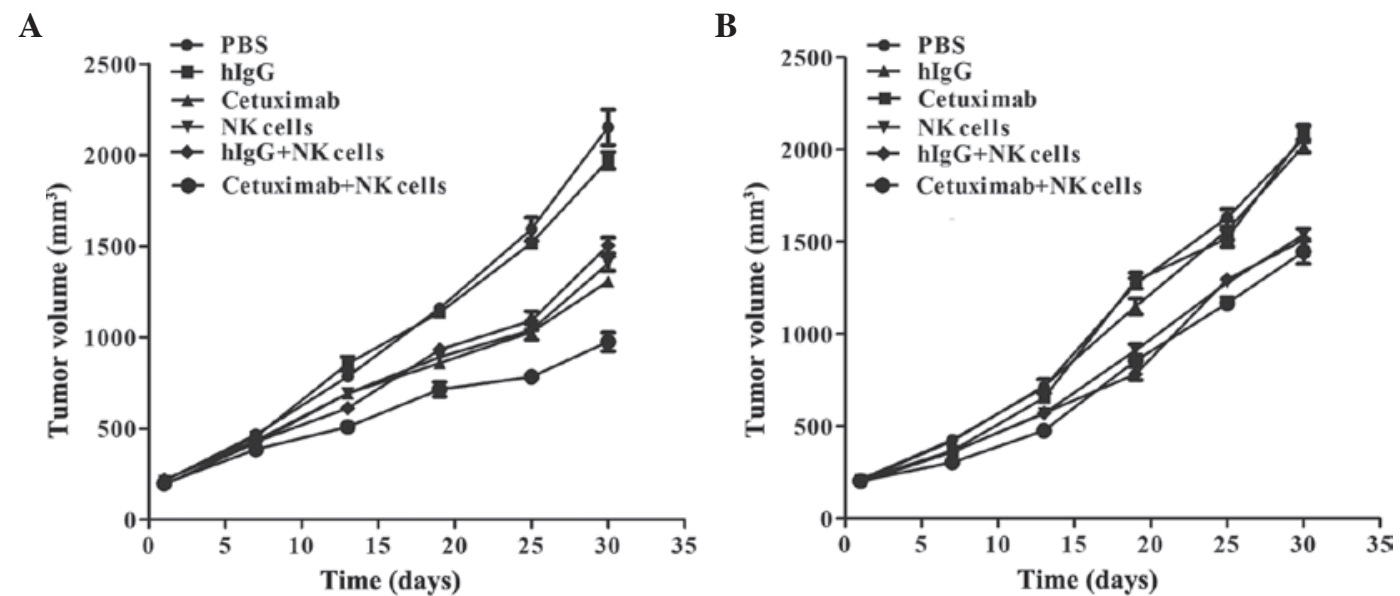

Figure 2. Tumor growth curves of (A) LOVO and (B) SW620 xenografts. PBS, phosphate-buffered saline; hIgG, human immunoglobulin G; NK, natural killer.

effect towards the LOVO and SW620 cells, and the inhibitory rate was positively associated with the effector-to-target ratios ( $r=0.994, P=0.006 ; r=0.972, P=0.028)$. In addition, the inhibitory rate was greater in the LOVO cells compared with the SW620 cells $(\mathrm{P}=0.003)$.

Mouse weight and xenograft tumor volume prior to treatment. After 5 days of subcutaneous implantation, the xenograft tumors grew well in the nude mice, and the tumor size grew to $\sim 200 \mathrm{~mm}^{3}$ after 14 days. For each group of mice, the weight and xenograft tumor volume was analyzed prior to treatment, and no statistically significant difference was shown $(\mathrm{P}=0.132$ and $\mathrm{P}=0.880$, respectively).

Xenograft tumor growth curve. The tumor growth curve showed that the LOVO xenograft underwent a growth restriction following the injection of cetuximab plus NK cells, and the average tumor volume was evidently decreased compared with other groups $(\mathrm{P}<0.0001$ compared with the $\mathrm{PBS}$ group, $\mathrm{P}<0.0001$ compared with the hIgG group, $\mathrm{P}=0.004$ compared with the cetuximab group, $\mathrm{P}=0.02$ compared with the $\mathrm{NK}$ cells group and $\mathrm{P}=0.01$ compared with the hIgG plus NK cells group). Furthermore, the tumor growth was also inhibited in the cetuximab only, NK cells only and NK cells plus hIgG groups, with similar inhibitory effects in each $(\mathrm{P}=0.113)$. The tumor volume was inhibited, but no statistically significantly difference was found, among the cetuximab plus NK cells, NK cells and NK cells plus hIgG groups of the SW620 xenograft models $(\mathrm{P}=0.374)$, and tumor growth was not inhibited in the cetuximab group (Fig. 2).

Xenograft tumor weight. At the end of experiment, the average LOVO xenograft tumor weight of the cetuximab plus NK cells group was decreased compared with any of the other groups $(\mathrm{P}<0.0001$ compared with the PBS group, $\mathrm{P}<0.0001$ compared with the hIgG group, $\mathrm{P}=0.004$ compared with the cetuximab group, $\mathrm{P}=0.047$ compared with the $\mathrm{NK}$ cells group and $\mathrm{P}=0.005$ compared with the $\mathrm{hIgG}$ plus $\mathrm{NK}$ cells group), and the inhibition rate was $47.92 \%$, which was $\sim 60 \%$ increased compared with the cetuximab or NK cells groups. The cetuximab, NK cells and NK cells plus hIgG groups also demonstrated an antitumor effect compared with $\mathrm{PBS}$ group $(\mathrm{P}=0.0002, \mathrm{P}=0.0004$ and $\mathrm{P}=0.0004$, respectively), but their tumor weights were increased compared with combination group $(\mathrm{P}=0.014, \mathrm{P}=0.017$ and $\mathrm{P}=0.023$, respectively). In SW620 xenograft models, the tumor weight showed no statistical differences among the cetuximab plus NK cell group, NK cells group and NK cells plus hIgG group $(\mathrm{P}=0.299)$, but their tumor weights were decreased compared the control group $(\mathrm{P}=0.008, \mathrm{P}=0.004$ 
A

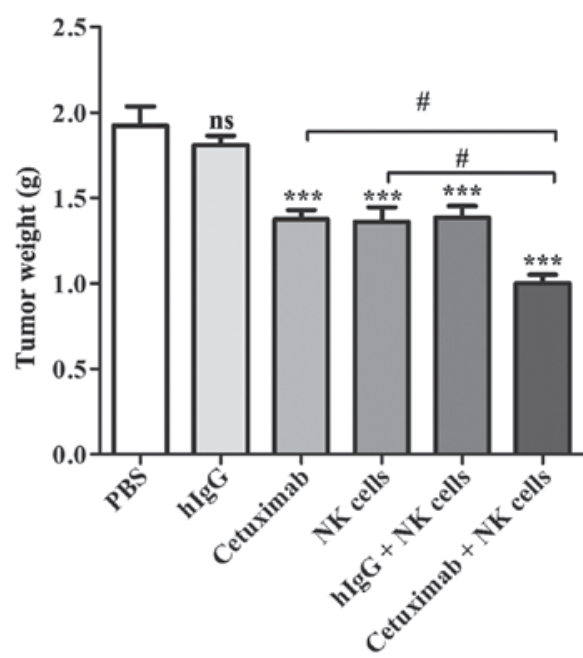

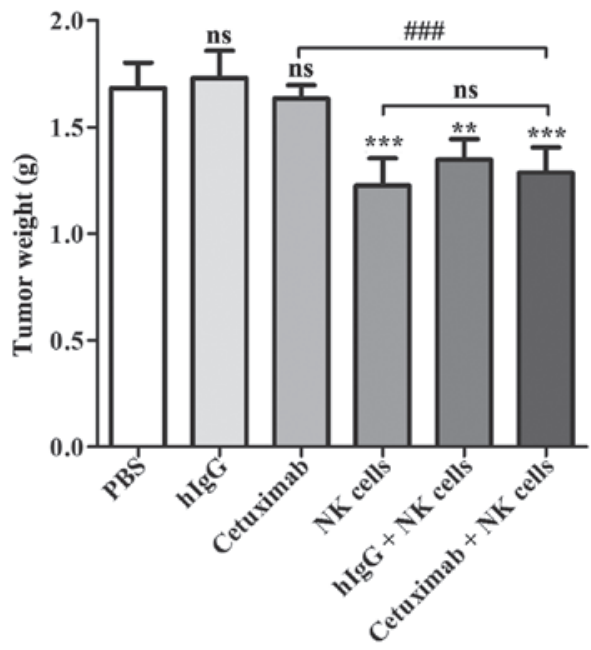

Figure 3. Tumor weights of (A) LOVO and (B) SW620 xenografts. Differences between groups were determined by the Bonferroni test. ${ }^{\text {ns }} \mathrm{P}>0.05$, ${ }^{* *} \mathrm{P}<0.01$ and ${ }^{* * * *} \mathrm{P}<0.001$ compared with the PBS-treated control group (top of bar); ${ }^{\text {ns }} \mathrm{P}>0.05,{ }^{\#} \mathrm{P}<0.05$ and ${ }^{\# \# \#} \mathrm{P}<0.001$ compared with the cetuximab plus NK cells group (line connecting bars). PBS, phosphate-buffered saline; hIgG, human immunoglobulin G; NK, natural killer.
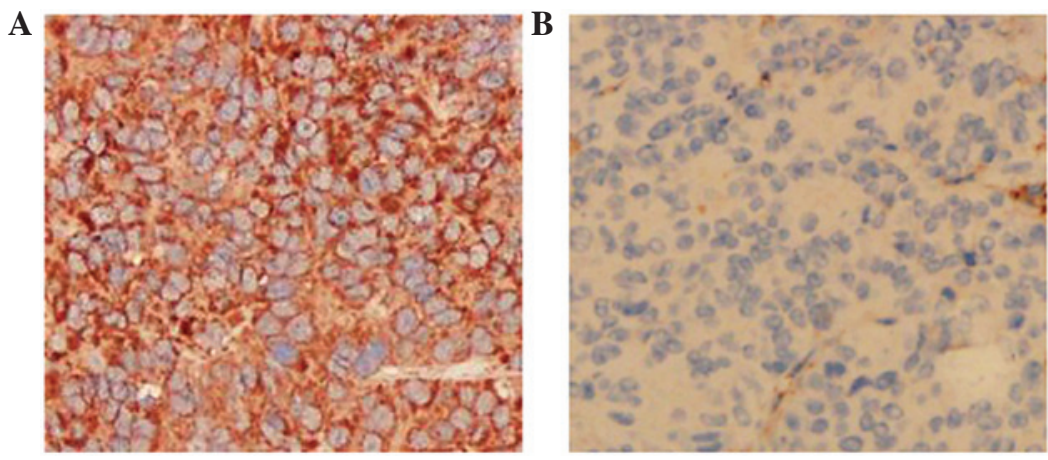

Figure 4. Expression of EGFR in xenograft tissues by immunohistochemistry. Original magnification, x200. (A) Positive (+++) expression of EGFR in LOVO xenografts. (B) Negative (-) expression of EGFR in SW620 xenografts. EGFR, epidermal growth factor receptor.
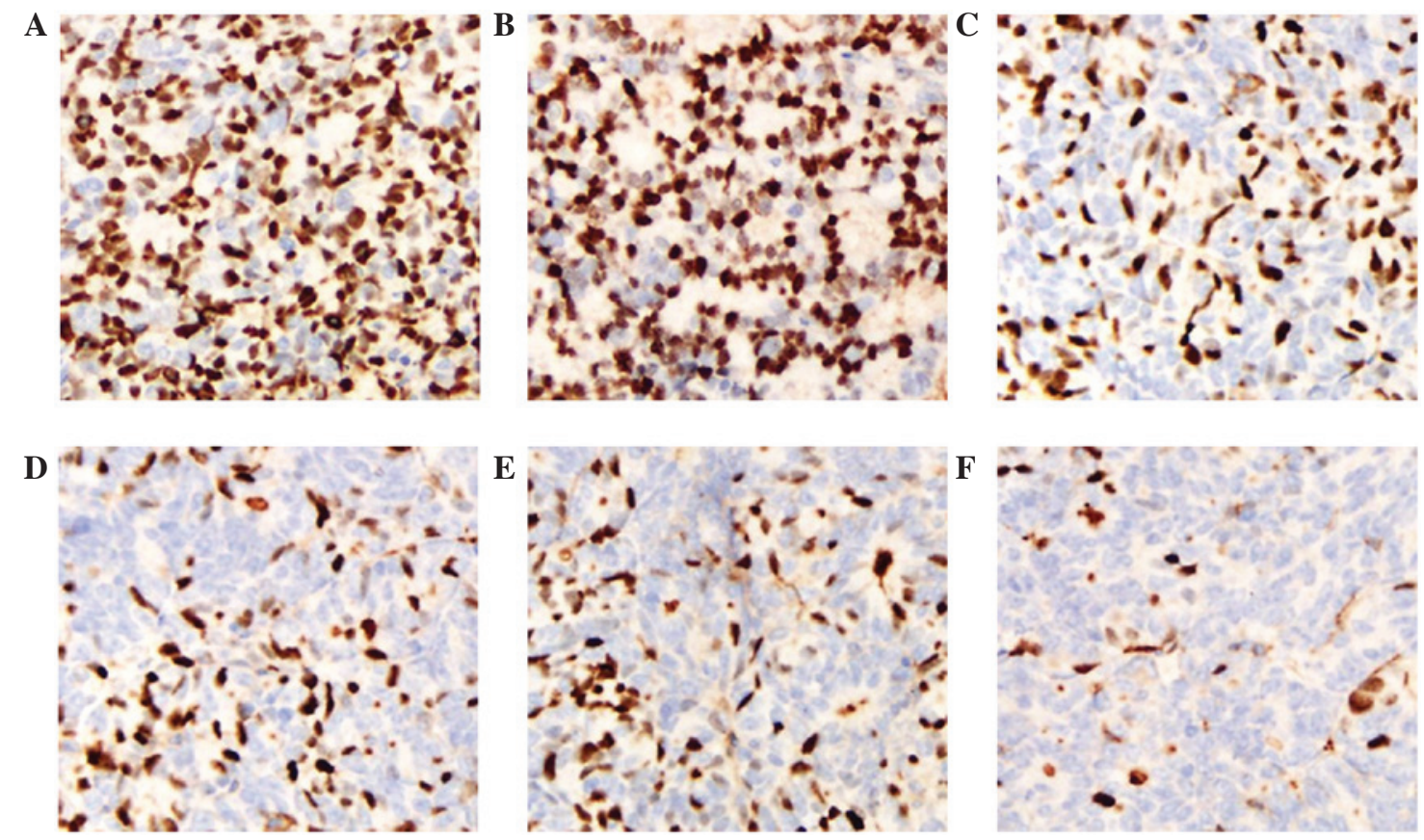

Figure 5. Expression of Ki-67 in LOVO xenograft tissues by immunohistochemistry. Original magnification, x200. (A) Phosphate-buffered saline group. (B) hIgG group. (C) Cetuximab group. (D) NK cells group. (E) hIgG+NK cells group. (F) Cetuximab+NK cells group. hIgG, human immunoglobulin G; NK, natural killer. 

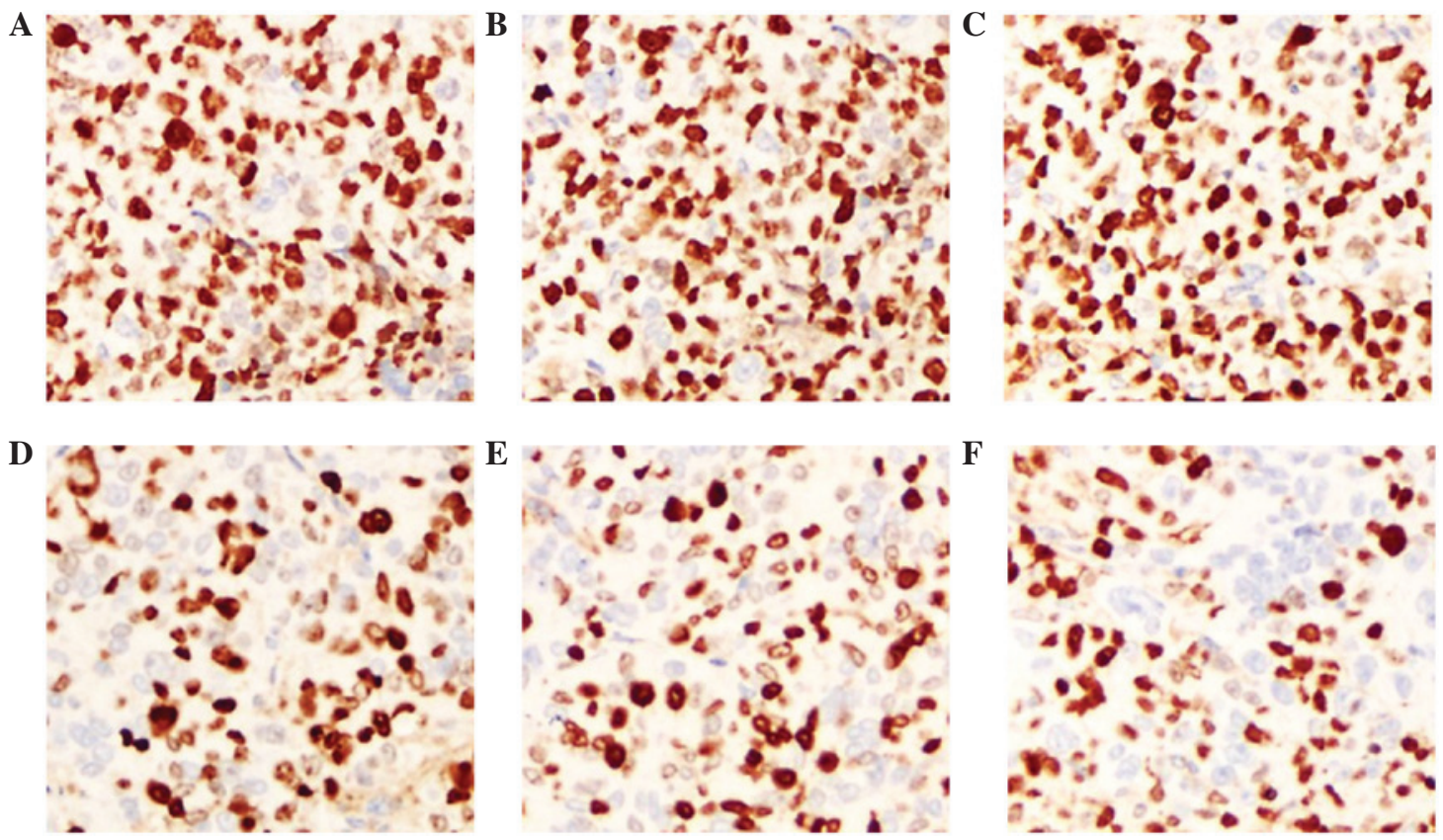

Figure 6. Expression of Ki-67 in SW620 xenograft tissues by immunohistochemistry. Original magnification, x200. (A) Phosphate-buffered saline group. (B) hIgG group. (C) Cetuximab group. (D) NK cells group. (E) hIgG+NK cells group. (F) Cetuximab+NK cells group. hIgG, human immunoglobulin G; NK, natural killer.
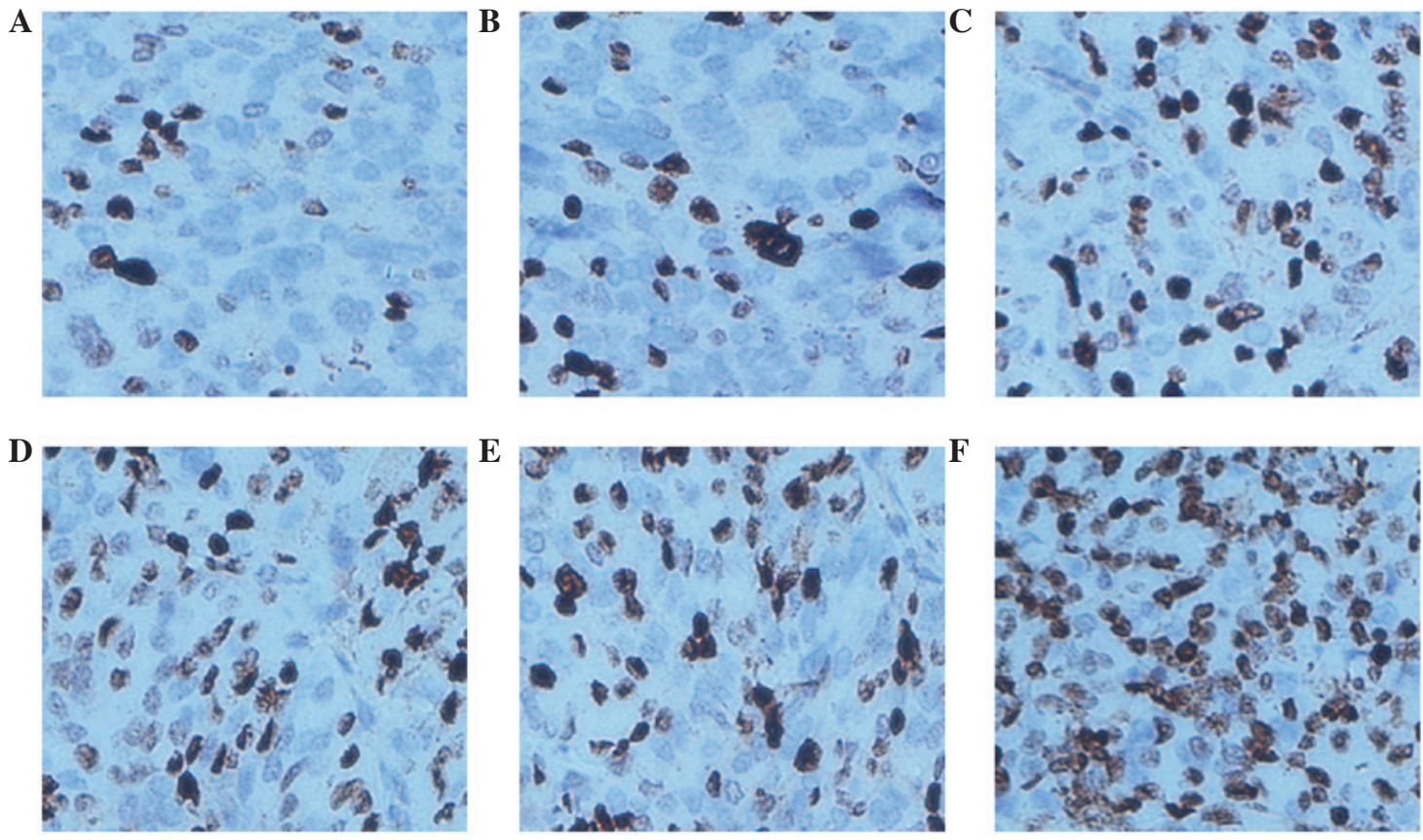

Figure 7. Apotosis in LOVO xenograft tissues by terminal deoxynucleotidyl transferase dUTP nick end labeling. Original magnification, $\mathrm{x} 200$. (A) Phosphatebuffered saline group. (B) hIgG group. (C) Cetuximab group. (D) NK cells group. (E) hIgG+NK cells group. (F) Cetuximab+NK cells group. hIgG, human immunoglobulin G; NK, natural killer.

and $\mathrm{P}=0.001$, respectively). Tumor weight was not affected by cetuximab only in the SW620 cell xenograft models $(\mathrm{P}=0.430$; Fig. 3; Table II).

EGFR staining. As shown in Fig. 4, IHC staining showed that EGFR was mainly expressed in the tumor cell membrane. The EGFR expression in the LOVO xenograft models was strong positive (+++), whereas the expression in the SW620 xenograft models was negative (-).
$\mathrm{Ki}-67$ staining. $\mathrm{Ki}-67$ is an indicator of cell proliferation and is mainly expressed in the cell nucleus. IHC staining showed that the Ki-67 expression of LOVO xenograft tumors in the cetuximab plus NK cells group was $23.8 \pm 3.89 \%$, which was decreased compared with the NK cells and cetuximab groups $(\mathrm{P}=0.003$ and $\mathrm{P}=0.002$, respectively). The expression of Ki-67 in the cetuximab plus NK cells group was weak positive $(+)$, whereas the expression in the control groups (PBS and hIgG) was strong positive $(+++)$. In the SW620 xenograft tumor 

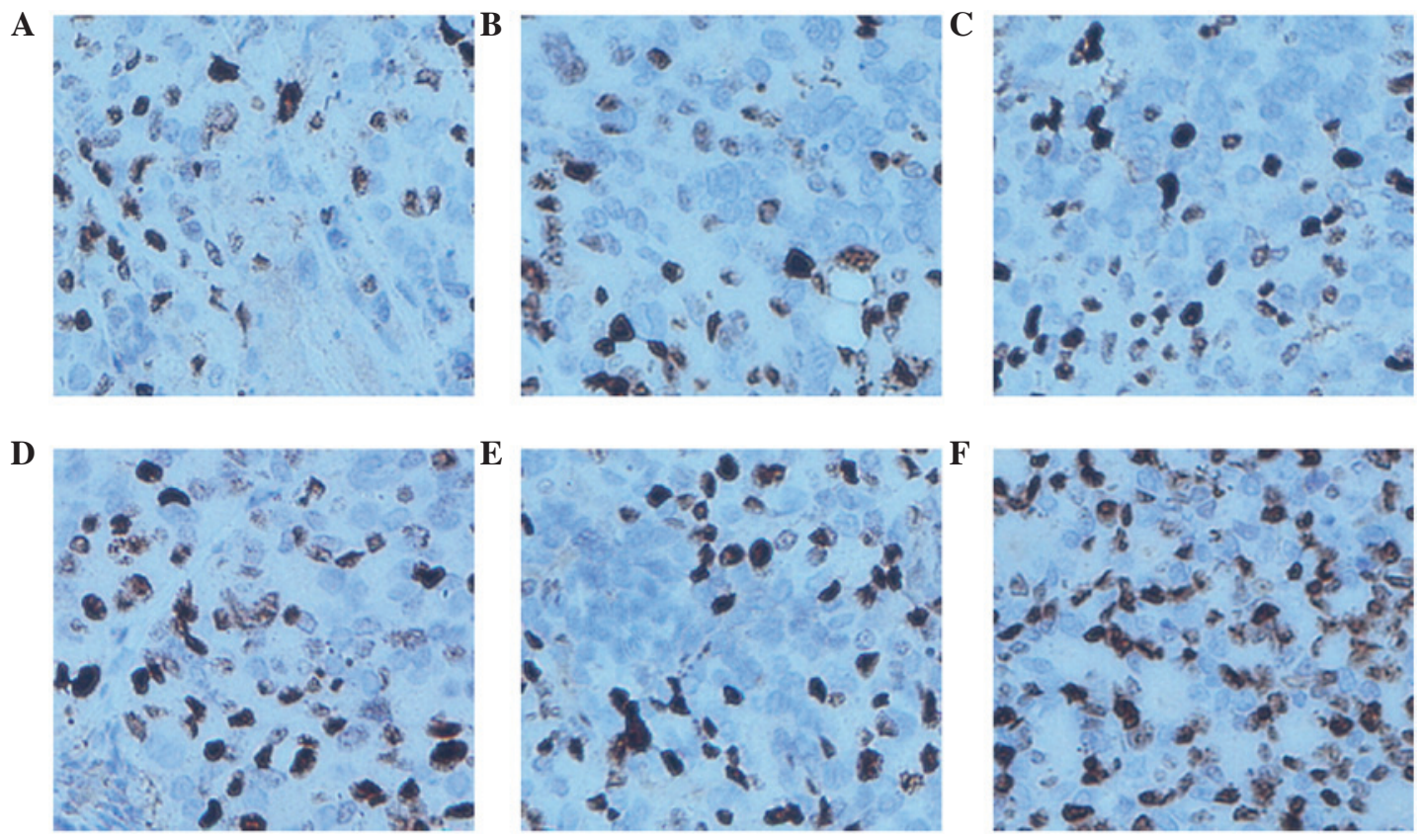

Figure 8. Apotosis in SW620 xenograft tissues by terminal deoxynucleotidyl transferase dUTP nick end labeling. Original magnification, x200. (A) Phosphatebuffered saline group. (B) hIgG group. (C) Cetuximab group. (D) NK cells group. (E) hIgG+NK cells group. (F) Cetuximab+NK cells group. hIgG, human immunoglobulin G; NK, natural killer.

Table III. Labeling index of Ki-67 in xenografts.

\begin{tabular}{lcc}
\hline & \multicolumn{2}{c}{ Ki-67 labelling index, \% } \\
\cline { 2 - 3 } Group & $\begin{array}{c}\text { LOVO } \\
\text { xenografts }\end{array}$ & $\begin{array}{c}\text { SW620 } \\
\text { xenografts }\end{array}$ \\
\hline Phosphate-buffered saline & $85.40 \pm 1.72$ & $83.80 \pm 4.14$ \\
hIgG & $83.80 \pm 2.27$ & $88.40 \pm 1.12$ \\
Cetuximab & $55.00 \pm 5.89$ & $83.00 \pm 1.67$ \\
NK cells & $52.20 \pm 5.49$ & $66.60 \pm 4.08$ \\
hIgG+NK cells & $54.00 \pm 7.07$ & $67.40 \pm 3.01$ \\
Cetuximab+NK cells & $23.80 \pm 3.89$ & $59.20 \pm 2.97$ \\
\hline
\end{tabular}

Values presented as mean \pm standard error of the mean. NK, natural killer; hIgG, human immunoglobulin G.

Table IV. Apoptosis index in xenografts.

\begin{tabular}{lcc}
\hline & \multicolumn{2}{c}{ Apoptosis index, \% } \\
\cline { 2 - 3 } Group & $\begin{array}{c}\text { LOVO } \\
\text { xenografts }\end{array}$ & $\begin{array}{c}\text { SW620 } \\
\text { xenografts }\end{array}$ \\
\hline Phosphate-buffered saline & $24.20 \pm 1.46$ & $25.40 \pm 2.91$ \\
hIgG & $23.40 \pm 2.91$ & $20.00 \pm 1.52$ \\
Cetuximab & $50.60 \pm 4.91$ & $24.20 \pm 2.96$ \\
NK cells & $50.80 \pm 4.97$ & $49.60 \pm 6.14$ \\
hIgG+NK cells & $47.40 \pm 3.47$ & $46.60 \pm 4.31$ \\
Cetuximab+NK cells & $73.80 \pm 4.35$ & $54.00 \pm 2.55$
\end{tabular}

Values presented as mean \pm standard error of the mean. NK, natural killer; hIgG, human immunoglobulin G. models, the Ki-67 expression in the cetuximab plus NK cells group was not decreased compared with the NK cells group $(\mathrm{P}=0.173)$. In addition, the Ki-67 expression in the cetuximab group also showed no significant difference compared with the control group $(\mathrm{P}=0.862)$. These results indicate that cetuximab or adoptive NK cells may inhibit the proliferation of tumor cells in LOVO xenografts, and that the combination of cetuximab and NK cells significantly improved this effect. Nevertheless, cell proliferation remained active and was not affected by cetuximab in the SW620 cell xenograft tumors (Figs. 5 and 6; Table III).

TUNEL assay. TUNEL assays are an indicator of cell apoptosis, in which the nuclei are stained brown in positively staining cells. The results showed that the AI of the LOVO xenograft tumors was $73.80 \pm 4.35 \%$ in the cetuximab combined with NK cells group, which was evidently increased compared with other groups $(\mathrm{P}<0.0001$ compared with the PBS group, $\mathrm{P}<0.0001$ compared with the $\mathrm{hIgG}$ group, $\mathrm{P}=0.008$ compared with the cetuximab group, $\mathrm{P}=0.008$ compared with the NK cells group and $\mathrm{P}=0.002$ compared with the $\mathrm{hIgG}$ plus $\mathrm{NK}$ cells group). The AI in the cetuximab, NK cells and NK cells plus hIgG groups increased compared with the PBS control group $(\mathrm{P}=0.0009, \mathrm{P}=0.0009$ and $\mathrm{P}=0.0003$, respectively). The $\mathrm{AI}$ of the SW620 xenograft tumors in the cetuximab combined with NK cells, the NK cells and the NK cells plus hIgG groups were increased compared with the PBS control group $(\mathrm{P}=0.018$, $\mathrm{P}=0.004$ and $\mathrm{P}=0.0007$, respectively); however no significant difference was identified between the 3 (non-control) groups $(\mathrm{P}=0.533)$. The $\mathrm{AI}$ in the cetuximab group was similar to the control group $(\mathrm{P}=0.780)$. These results suggest that $\mathrm{NK}$ cells may promote LOVO xenograft tumor cell apoptosis in vivo, and that the effects are increased by the participation of cetuximab. However, SW620 xenograft tumor cell apoptosis was not influenced by cetuximab (Figs. 7 and 8; Table IV). 


\section{Discussion}

The present preclinical study has focused on the ADCC activity of adoptive NK cells in combination with cetuximab in nude mice xenograft models. The results suggested that NK cells alone could inhibit tumor growth in the LOVO and SW620 xenografts. Following combination with cetuximab, NK cells had a stronger inhibiting effect on the growth of LOVO xenografts compared with NK cell therapy or cetuximab therapy. However, adding cetuximab to NK cells did not enhance the ADCC activity towards SW620 xenografts. Pathological changes demonstrated that the varied EGFR expression levels of the two tumor cells were responsible for these conflicting results.

NK cells, discovered $\sim 40$ years ago, are considered to be the most effective cytotoxic lymphocytes for counteracting cancer (25). Mandal and Viswanathan (25) reported that adoptive NK cell transfer was applied to advanced CRC patients that were refractory to standard therapy, and was demonstrated to be efficacious and safe. The data from the present study also verified that the adoptive injection of NK cells can inhibit LOVO or SW620 xenograft tumor cell proliferation and promote apoptosis compared with a PBS control. However, the effect was not particularly strong and the tumor volume and weight remained at a relatively high level.

Cetuximab has been approved for the first-line treatment of EGFR-positive metastatic CRC and somewhat demonstrates therapeutic efficacy when used as a monotherapy in metastatic CRC patients for which chemotherapy has failed. In the present study, cetuximab alone also showed considerable antitumor activity in LOVO xenografts compared with the $\mathrm{hIgG}$ control group, and had a similar effect to NK cells. However, cetuximab did not show a significant tumor inhibitory effect in the SW620 xenografts.

The therapeutic target of cetuximab is EGFR, which is a transmembrane glycoprotein (molecular weight, $170 \mathrm{KD}$ ) that is composed by extracellular, transmembrane and intracellular tyrosinekinase domains. EGFR is widely expressed in human cell membranes and highly expressed by a variety of epithelial tumor cells, including $60-80 \%$ CRCs (26). The overexpression of EGFR will lead to uncontrolled cell growth and proliferation $(3,27,28)$, which can result in disease progression, metastasis and recurrence and is associated with a poor outcome of CRC (10). Using IHC staining, the present study confirmed that the LOVO cells were EGFR-positive (+++) and that the SW620 cells were EGFR-negative (-), which was consistent with in vitro experiments reported in the literature $(12,29)$. This finding indicated that LOVO or SW620 cells transplanted into nude mice retained the EGFR-positive or -negative biological characteristics. Seo et al (30) verified that cetuximab-mediated ADCC activity was strongly and significantly correlated with the cell surface expression level of EGFR. After completely binding to EGFR, cetuximab can inhibit of the downstream signals induced by its natural ligands, epidermal growth factor and transforming growth factor- $\alpha$, exposing the cancer cells to the to immune system (12). NK cells can then lyse the tumor cells bound to cetuximab via ADCC activity (12). Furthermore, the extent of the activation of NK cells by cetuximab has also been found to correlate with the level of EGFR expression (12). Therefore, EGFR-negative SW620 xenografts did not respond to cetuximab and adding cetuximab to NK cells did not enhance the tumor inhibitory effect of adoptive NK cells in the present study.

The combination of cetuximab with irinotecan, fluorouracil (5-FU) and folinic acid chemotherapy or oxaliplatin, 5-FU and folinic acid chemotherapy was shown to be effective in a previous study (10), yet conflicting results were found in the COIN (31) and NORDIC VII (32) trials when cetuximab was combined with oxaliplatin-based chemotherapy. One of the most important aspects was the adverse side effects of chemotherapy. Thus, the present study sought for a safer and more effective way utilize cetuximab for the treatment of metastatic CRC. Pahl et al (12) reported that cetuximab enhances the ADCC activity of NK cells towards osteosarcoma, and the present in vitro study demonstrates that cetuximab may significantly enhance NK-mediated ADCC activity in CRC cell lines. Therefore, cetuximab was combined with adoptive NK cells in vivo and, as expected, cetuximab was found to intensify the ADCC activity of adoptive NK cells towards LOVO xenografts. In agreement with previous studies $(10,33)$, the present study found that NK cell-mediated ADCC activity could be a crucial antitumor mechanism of cetuximab in vivo.

Previously, however, certain studies expressed diverging opinions. Wild et al (34) reported that the effect of cetuximab in vivo did not necessarily associate with EGFR expression on the target cell surface, or was affected by a variety of factors in vivo. Similarly, Kurai et al (9) claimed that even low EGFR expression was sufficient for maximum ADCC activity. These reports indicate that the ADCC activity is affected by numerous factors. The ultimate purpose of the present study is to improve therapeutic efficacy and decrease the toxicity of cetuximab or adoptive NK cells for advanced CRC; however, numerous problems remain to be solved, including the suitable amount of NK cells and the minimum dosage of cetuximab required to induce maximal anticancer ADCC activity. Thus, the specific clinical application of this combination also requires additional studies.

In conclusion, the present study suggests that cetuximab combined with adoptive NK cells may be a potential immunotherapy for advanced CRC patients with increased EGFR expression, particularly for patients that are not sensitive to isolated NK cell or cetuximab therapies. The expression of EGFR on tumor cells may be useful as a prediction index for evaluating the efficacy of the combination therapy. In addition, the present study elucidates a novel strategy for promoting cancer treatment by combining molecular target therapy with adoptive cell therapy; however, additional preclinical studies and clinical trials are required in order to focus on their efficacy in combination.

\section{Acknowledgements}

The present study was supported by the Medical Innovation Project of Fujian Province (Fujian, China; grant no. 2011-CX-17). The authors would like to thank Miss Qinying Liu for assistance in manipulating the figures, Miss Yangmei $\mathrm{Xu}$ for modification of the manuscript and the healthy donors that agree to be involved in the present study. 


\section{References}

1. Chen W, Zheng R, Zhang S, Zhao P, Zeng H and Zou X: Report of cancer incidence and mortality in China, 2010. Ann Transl Med 2: 61, 2014.

2. Siegel R, Ma J, Zou Z and Jemal A: Cancer statistics, 2014. CA Cancer J Clin 64: 9-29, 2014

3. Noguchi T, Ritter G and Nishikawa H: Antibody-based therapy in colorectal cancer. Immunotherapy 5: 533-545, 2013.

4. Kuppen PJ, Gorter A, Hagenaars M, Jonges LE, Giezeman-Smits KM, Nagelkerke JF, Fleuren G and van de Velde CJ: Role of NK cells in adoptive immunotherapy of metastatic colorectal cancer in a syngeneic rat model. Immunol Rev 184: 236-243, 2001.

5. Barkholt L, Alici E, Conrad R, Sutlu T, Gilljam M, Stellan B, Christensson B, Guven H, Björkström NK, Söderdahl G, et al: Safety analysis of ex vivo-expanded NK and NK-like T cells administered to cancer patients: A phase I clinical study. Immunotherapy 1: 753-764, 2009.

6. de Castro-Carpeño J, Belda-Iniesta C, Casado Sáenz E, Hernández Agudo E, Feliu Batlle J and González Barón M: EGFR and colon cancer: A clinical view. Clin Transl Oncol 10: 6-13, 2008.

7. Huether A, Höpfner M, Baradari V, Schuppan D and Scherübl H: EGFR blockade by cetuximab alone or as combination therapy for growth control of hepatocellular cancer. Biochem Pharmacol 70 1568-1578, 2005.

8. Troiani T, Zappavigna S, Martinelli E, Addeo SR, Stiuso P, Ciardiello F and Caraglia M: Optimizing treatment of metastatic colorectal cancer patients with anti-EGFR antibodies: Overcoming the mechanisms of cancer cell resistance. Expert Opin Biol Ther 13: 241-255, 2013.

9. Kurai J, Chikumi H, Hashimoto K, Yamaguchi K, Yamasaki A, Sako T, Touge H, Makino H, Takata M, Miyata M, et al: Antibody-dependent cellular cytotoxicity mediated by cetuximab against lung cancer cell lines. Clin Cancer Res 13: 1552-1561, 2007.

10. Sotelo Lezama MJ, Sastre Valera J and Díaz-Rubio García E: Impact of cetuximab in current treatment of metastatic colorectal cancer. Expert Opin Biol Ther 14: 387-399, 2014.

11. Bachanova V and Miller JS: NK cells in therapy of cancer. Crit Rev Oncog 19: 133-141, 2014.

12. Pahl JH, Ruslan SE, Buddingh EP, Santos SJ, Szuhai K, Serra M, Gelderblom H, Hogendoorn PC, Egeler RM, Schilham MW and Lankester AC: Anti-EGFR antibody cetuximab enhances the cytolytic activity of natural killer cells toward osteosarcoma. Clin Cancer Res 18: 432-441, 2012.

13. Kohrt HE, Colevas AD, Houot R, Weiskopf K, Goldstein MJ, Lund P, Mueller A, Sagiv-Barfi I, Marabelle A, Lira R, et al: Targeting CD137 enhances the efficacy of cetuximab. J Clin Invest 124: 2668-2682, 2014.

14. Cheng M, Chen Y, Xiao W, Sun R and Tian Z: NK cell-based immunotherapy for malignant diseases. Cell Mol Immunol 10: 230-252, 2013

15. Shiokawa M, Takahashi T, Murakami A, Kita S, Ito M, Sugamura K and Ishii N: In vivo assay of human NK-dependent ADCC using NOD/SCID/gamma(null) (NOG) mice. Biochem Biophys Res Commun 399: 733-737, 2010.

16. Lee SC, Srivastava RM, López-Albaitero A, Ferrone S and Ferris RL: Natural killer (NK): Dendritic cell (DC) cross talk induced by therapeutic monoclonal antibody triggers tumor antigen-specific T cell immunity. Immunol Res 50: 248-254, 2011.

17. Ogbomo H, Cinatl J Jr, Mody CH and Forsyth PA: Immunotherapy in gliomas: Limitations and potential of natural killer (NK) cell therapy. Trends Mol Med 17: 433-441, 2011.

18. Ottaiano A, Scala S and Iaffaioli VR: Cetuximab-dependent ADCC in cancer: Dream or reality? Cancer Immunol Immunother 59: 1607-1608, 2010.
19. Qu YH and Li Y: Progress of study on antitumor effects of antibody dependent cell mediated cytotoxicity-review. Zhongguo Shi Yan Xue Ye Xue Za Zhi 18: 1370-1375, 2010 (In Chinese).

20. Srivastava RM, Lee SC, Andrade Filho PA, Lord CA, Jie HB, Davidson HC, López-Albaitero A, Gibson SP, Gooding WE, Ferrone S and Ferris RL: Cetuximab-activated natural killer and dendritic cells collaborate to trigger tumor antigen-specific T-cell immunity in head and neck cancer patients. Clin Cancer Res 19: 1858-1872, 2013.

21. Balasa B, Yun R, Belmar NA, Fox M, Chao DT, Robbins MD, Starling GC and Rice AG: Elotuzumab enhances natural killer cell activation and myeloma cell killing through interleukin-2 and TNF- $\alpha$ pathways. Cancer Immunol Immunother 64: 61-73, 2015.

22. Roda JM, Joshi T, Butchar JP, McAlees JW, Lehman A, Tridandapani S and Carson WE III: The activation of natural killer cell effector functions by cetuximab-coated, epidermal growth factor receptor-positive tumor cells is enhanced by cytokines. Clin Cancer Res 13: 6419-6428, 2007.

23. JD H, JW Y, RM C, MG Y and QH Z: The ADCC function of NK cells combined with cetuximab on colon cancer cell. Fu Jian Yi Ke Da Xue Xue Bao 46: 20-27, 2012 (In Chinese).

24. Yang X, Zhang X, Mortenson ED, Radkevich-Brown O, Wang Y and Fu YX: Cetuximab-mediated tumor regression depends on innate and adaptive immune responses. Mol Ther 21: 91-100, 2013.

25. Mandal A and Viswanathan C: Natural killer cells: In health and disease. Hematol Oncol Stem Cell Ther 8: 47-55, 2015.

26. Vincenzi B, Zoccoli A, Pantano F, Venditti O and Galluzzo S: Cetuximab: From bench to bedside. Curr Cancer Drug Targets 10: 80-95, 2010.

27. Baselga J: Why the epidermal growth factor receptor? The rationale for cancer therapy. Oncologist 7 (Suppl 4): S2-S8, 2002.

28. Goldberg RM and Gill S: Recent phase III trials of fluorouracil, irinotecan, and oxaliplatin as chemotherapy for metastatic colorectal cancer. Cancer Chemother Pharmacol 54 (Suppl 1): S57-S64, 2004.

29. Holubec L, Liska V, Matejka VM, Fiala O, Dreslerova J, Mrazkova P, Treska V and Finek J: The role of cetuximab in the treatment of metastatic colorectal cancer. Anticancer Res 32: 4007-4011, 2012.

30. Seo Y, Ishii Y, Ochiai H, Fukuda K, Akimoto S, Hayashida T, Okabayashi K, Tsuruta M, Hasegawa $\mathrm{H}$ and Kitagawa Y: Cetuximab-mediated ADCC activity is correlated with the cell surface expression level of EGFR but not with the KRAS/BRAF mutational status in colorectal cancer. Oncol Rep 31: 2115-2122, 2014.

31. Maughan TS, Adams RA, Smith CG, Meade AM, Seymour MT, Wilson RH, Idziaszczyk S, Harris R, Fisher D, Kenny SL, et al; MRC COIN Trial Investigators: Addition of cetuximab to oxaliplatin-based first-line combination chemotherapy for treatment of advanced colorectal cancer: Results of the randomised phase 3 MRC COIN trial. Lancet 377: 2103-2114, 2011.

32. Tveit KM, Guren T, Glimelius B, Pfeiffer P, Sorbye H, Pyrhonen S, Sigurdsson F, Kure E, Ikdahl T, Skovlund E, et al: Phase III trial of cetuximab with continuous or intermittent fluorouracil, leucovorin, and oxaliplatin (Nordic FLOX) versus FLOX alone in first-line treatment of metastatic colorectal cancer: The NORDIC-VII study. J Clin Oncol 30: 1755-1762, 2012.

33. Li S, Schmitz KR, Jeffrey PD, Wiltzius JJ, Kussie P and Ferguson KM: Structural basis for inhibition of the epidermal growth factor receptor by cetuximab. Cancer Cell 7: 301-311, 2005.

34. Wild R, Fager K, Flefleh C, Kan D, Inigo I, Castaneda S, Luo FR, Camuso A, McGlinchey K and Rose WC: Cetuximab preclinical antitumor activity (monotherapy and combination based) is not predicted by relative total or activated epidermal growth factor receptor tumor expression levels. Mol Cancer Ther 5: 104-113, 2006. 